\title{
Power Detectors for Integrated Microwave/mm-Wave Imaging Systems in Mainstream Silicon Technologies
}

\author{
Qun Jane Gu, James C. Li , and Adrian Tang
}

\begin{abstract}
This paper analyzes and compares three different types of detectors, including CMOS power detectors, bipolar power detectors, and super-regenerative detectors, deployed in the literature for integrated microwave/mm-wave imaging systems in mainstream silicon technologies. Each detector has unique working mechanism and demonstrates different behavior with respects to bias conditions, input signal power, as well as bandwidth responses. Two Figure-of-Merits for both wideband and narrowband imaging have been defined to quantify the detector performance comparison. CMOS and Bipolar detectors are good for passive imaging, while super regenerative detectors are superior for active imaging. The analytical results have been verified by both simulation and measurement results. These analyses intend to provide design insights and guidance for integrated microwave/mm-wave imaging power detectors.
\end{abstract}

Index Terms - Bipolar, CMOS, Detector, Imager, Super-Regenerative.

\section{INTRODUCTION}

$\mathrm{M}$ icrowave/mm-wave systems in silicon have been widely investigated due to its high potentials in different applications $[1,2,3]$. In particular, microwave/mm-wave imaging systems, including both passive and active imaging, have been employed broadly in different applications, from space imaging, security check to noninvasive biomedical diagnosis. In particular, many biologic imaging applications emerge recently due to the need to investigate the working mechanisms of biological bodies $[4,5]$. A power based detector serves as one of the key components in many of these imaging systems, and often determines the system performance. So far, many different devices and circuit architectures have been disclosed to conduct power detection in the literature. To facilitate wide deployment, detectors compatible with mainstream processes are preferred to be naturally integrated into imaging systems with small form factors [6-21], which will be the focus of this paper. Among them, there are three major types of detectors. In silicon CMOS technologies, square root power detection has been developed [6-8], which utilizes the nonlinear I-V characteristics of MOS transistors and demonstrates a decent performance in mm-wave regime. In

Q. Jane $\mathrm{Gu}$ is with the University of California, Davis, CA 9561, USA (e-mail: jgu@ucdavis.edu), James. C. Li is with HRL Laboratories, LLC, Malibu, CA, 90265, USA (e-mail: jli@hrl.com), and Adrian Tang is with Jet Propulsion Laboratory, Pasadena, CA 91109, USA (e-mail: Adrian.J.Tang@jpl.nasa.gov) contrast, bipolar or diode transistors often demonstrate a high transconductance as well as a more nonlinear I-V characteristic, therefore, can deliver a high power detection gain. Because of the high responsivity, a large portion of existing imaging applications employ integrated bipolar detectors/diodes in technologies, such as SiGe, GaAs, and InP [9-14]. In additional, to allow the best performance from each technology, silicon based heterogeneous technologies have been investigated. For example, one research aims to integrate InP HBT with silicon CMOS, which has been supported by the US DARPA COSMOS (Compound Semiconductor Materials on Silicon) program. The success of the heterogeneous integration will open new opportunities and support circuit and system performance which may not be possible by any single individual process. This paper also includes an InP HBT based detector in a heterogeneous process as an example to investigate the bipolar type detector. Super-regenerative circuits were invented by Armstrong in 1912 and used to dominate radio market until the 1930s when FM became popular. Super-regenerative receivers demonstrate low power consumption and high frequency operations. Most recently, it has been found that the super-regenerative circuit architecture can offer extremely high gain for power detection and has been demonstrated successfully in ultra-low power applications [15-21].

All of the three detector types have been reported in the literature. However, no detail comparison of them have been conducted to the authors' best knowledge. There are still lack of systematic analyses on how to choose a detector architecture and optimize it for different applications based upon its working mechanisms, with the consideration of different bandwidth requirements, the input signal power range, as well as the imager system integration concerns. This paper intends to provide a systematic and theoretical comparison among the three detectors, discuss the corresponding optimization schemes, and support quantitative comparison by the newly defined FOMs for different applications. In addition, this paper points out that the detector performance can be significantly affected by the input power, which will affect their employment in different scenarios. It also for the first time presents a procedure to optimize an integrated detector and shed light on circuit optimization and architecture selection for different imaging systems.

The paper is organized as follows. Section II introduces the key merits of power detectors for imaging systems. 
Section III discusses the working mechanisms of the two device based detectors: CMOS and bipolar detectors. Section IV provides a design guidance and procedure on how to integrate the detectors into an imager system. Section V illustrates the super regenerative detector, originally proposed by the authors, which complements the basic device based detectors to save power hungry preceding amplifiers with high responsivity and low power consumption. Section VI presents the measurement results of three individual detectors and comparison summary, followed by the conclusions in Section VII.

\section{Key Merits of Detectors For IMAGING Systems}

Two different types of imaging systems, active and passive imaging systems, are often used. Active imaging systems detect objects by sensing the signal's strength or phase, which is transmitted through the object from a dedicated illumination source or reflected back from the object. The illuminator is usually included in active image systems [15]. In contrast, a passive imaging system does not have a dedicated illumination source. Instead, the passive imaging system relies on the radiation from the object itself, such as black body radiation, or the reflection due to other illumination sources not belonging to the imaging systems to observe the object and construct its image. Therefore, a passive imaging system often receives much lower power input signals compared with an active imaging system. Nonetheless, both imaging systems share similar system specifications to characterize their performances. First, responsivity is used to quantify the response strength of an imaging system, which is analogy to the gain definition in a communication receiver, and is defined as the ratio of the generated signal voltage or current over the incident signal power. Second, NEP (noise equivalent power), which is analogy to the input referred noise in a communication receiver, is defined as the signal power that delivers a unit signal-to-noise ratio at the output in one hertz bandwidth. Third, NETD (noise equivalent temperature difference), which is equivalent to the sensitivity in communication systems, determines the imager temperature resolution and is related to signal bandwidth and integration time. Fourth, input signal bandwidth that is similar to the bandwidth definition in communication systems determines the imager operating frequency range and also affects imaging systems' NETD. Among these specifications, responsivity and NEP are the two most critical specifications for power detectors in both active and passive imagers, while passive imagers need a much wider operational bandwidth compared with active imagers to minimize its NETD. In addition, the imagers expect different input power in different applications, which demands design trade-offs due to detector performance dependence on input power. The following analysis focuses on analyzing the responsivity and NEP of each type of detector, in particular, their relation to input power that has not been disclosed in detail in the literature to the authors' knowledge.

\section{COMParison OF BASIC POWER DETECTORS}

Two types of device based power detectors are widely used in integrated imager systems. One is CMOS power detectors, the other one is bipolar power detectors. Since diode based power detectors have similar I/V relationship and share the similar design considerations as bipolar ones, it is included into the bipolar detector category.

\section{A. CMOS Power Detector}

CMOS transistors can work in three different regions bearing dissimilar I-V characteristics, saturation, subthreshold, and triode regions, therefore leading to quite different detector performances in different regions. Among them, the triode

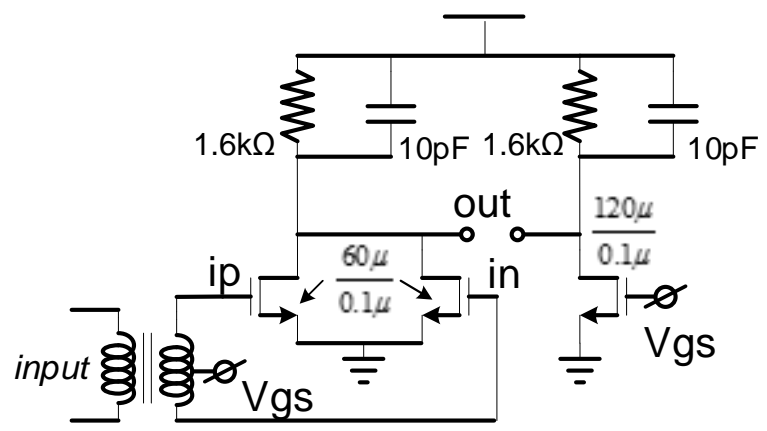

Fig. 1 A CMOS based detector in a $65 \mathrm{~nm}$ CMOS technology

region should be avoided due to a low responsivity, which roots from the linear relationship between input gate voltage and output drain current. In addition, the resistor like behavior of the CMOS transistor in the triode region cannot deliver a good NEP. Therefore, only the other two operation regions are discussed.

Fig. 1 presents a classical CMOS power detector, which has been implemented in a $65 \mathrm{~nm}$ technology, with the device $\mathrm{f}_{\mathrm{T}}$ about $200 \mathrm{GHz}$. This pseudo- differential power detector configuration shares an identical optimization scheme as a common source based single-ended power detector, except providing better noise immunity.

During the saturation region, the conventional square law model of MOS transistors describes its I-V relation:

$$
I_{s a t}=K_{1}\left(V_{g s 0}+v_{i n}-V_{T H}\right)^{2}
$$

where $K_{1}$ is device related coefficient, including the effects of device size, electron mobility, unit gate channel capacitance; $V_{g s o}$ is the biased gate source voltage, $V_{T H}$ is the device threshold voltage, and $v_{i n}$ is the input signal voltage. The power detection gain, defined as responsivity in imaging systems, is proportional to the second order derivation of the output current versus the input voltage, expressed as:

$$
\frac{\partial^{2} I_{\text {sat }}}{\partial v_{\text {in }}{ }^{2}}=2 K_{1} \quad R_{P}=2 K_{1} R
$$

where $\mathrm{R}$ is the drain load resistance. Eqn. (2) indicates that for long channel square-law governed devices, CMOS power detector responsivity is independent of device bias conditions.

In deep-scaled technologies, the transistors do not exactly follow the first order square law model, while the short-channel effects leading to the adjustment of the device I-V relation: 


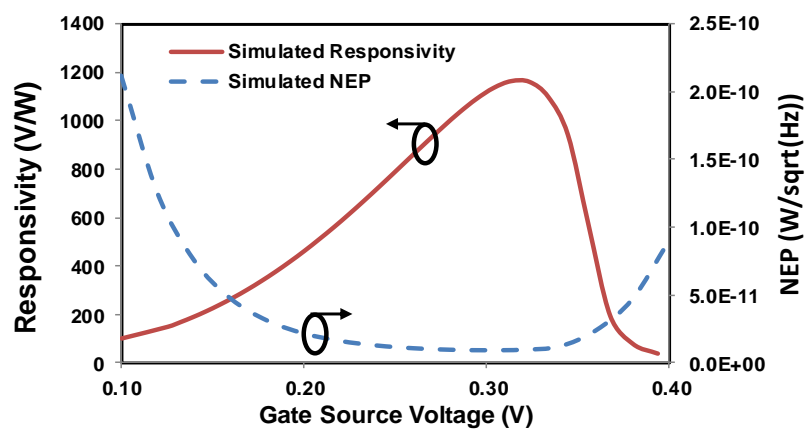

Fig. 2 Simulated responsivity (solid line) and NEP (dash line) versus CMOS gate source bias voltage of the CMOS detector in a $65 \mathrm{~nm}$ technology at $100 \mathrm{GHz}$

$$
I_{s a t}=K_{1} \frac{\left(V_{g s 0}-V_{T H}+v_{i n}\right)^{2}}{1+K_{2}\left(V_{g s 0}-V_{T H}+v_{i n}\right)}
$$

where $K_{1}$ and $K_{2}$ are process related constants. The device responsivity can be expressed as:

$$
R_{P}=\frac{\partial^{2} I_{s a t}}{\partial v_{i n}{ }^{2}} R=\frac{2 K_{1} R}{\left(1+K_{2}\left(V_{g s 0}-V_{T H}+v_{i n}\right)\right)^{2}}
$$

Eqn. (4) suggests that the responsivity of a CMOS power detector in the saturation region drops with the increase of the device overdrive voltage in deep-scaled technologies. In other words, the detector responsivity drops with device bias current $I_{s a t}$. When input power is small, i.e. $v_{i n} \ll V_{g s 0}-V_{T H}$, the responsivity remains relative constant. When input voltage swing is comparable with the device overdrive voltage, the responsivity decreases with increasing signal strength in deep-scaled technologies.

In the sub-threshold region, CMOS I-V relation can be expressed as:

$$
I_{s u b T}=I_{0} \exp \left(\frac{V_{g s 0}-V_{T H}+v_{i n}}{\xi V_{T}}\right)
$$

where $I_{0}$ is a constant current associated with device parameters, $\xi$ is a non-linear factor, and $V_{T}=k T / q$. The corresponding detector responsivity can be derived as

$$
R_{P}=\frac{\partial^{2} I_{s u b T}}{\partial v_{i n}{ }^{2}} R=\frac{I_{s u b T} R}{\left(\xi V_{T}\right)^{2}}
$$

Eqn (6) indicates that, in the subthreshold region, the CMOS power detector responsivity increases with the device bias current $I_{\text {subT }}$.

The opposite trends of the responsivity versus bias current indicated by Eqns. (4) and (6) imply that the maximum detector responsivity occurs when the CMOS detector devices are biased at the transition between subthreshold and saturation regions. That is, the gate source voltage is around the device threshold voltage. The simulated responsivity in Fig. 2 confirms that the maximum detector responsivity occurs around $300 \mathrm{mV}$, close to the device threshold voltage.

The NEP is defined by the ratio of the output voltage noise over the responsivity. With the output voltage noise represented by $V_{n}^{2}=4 K T R+4 K T \gamma g_{m} R^{2}$, the NEPs in both saturation and subthreshold regions can be formulated as:

$$
\begin{aligned}
N E P_{\text {sat }} & =\frac{\sqrt{4 K T R+4 K T \gamma g_{m} R^{2}}}{\left(2 K_{1} R\right) /\left(1+K_{2}\left(V_{g s 0}-V_{T H}+v_{i n}\right)\right)^{2}} \\
N E P_{\text {subT }} & =\frac{\sqrt{4 K T R+4 K T \gamma R^{2} I_{\text {subT }} /\left(\xi V_{T}\right)}}{\left(I_{\text {subT }} R\right) /\left(\xi V_{T}\right)^{2}}
\end{aligned}
$$

where $\mathrm{K}$ is the Boltzmann's constant, $\mathrm{g}_{\mathrm{m}}$ is device transconductance. Eqn. (7) indicates that the NEP increases with the gate source bias voltage in the saturation region. In contrast, Eqn. (8) suggests that NEP in the subthreshold region

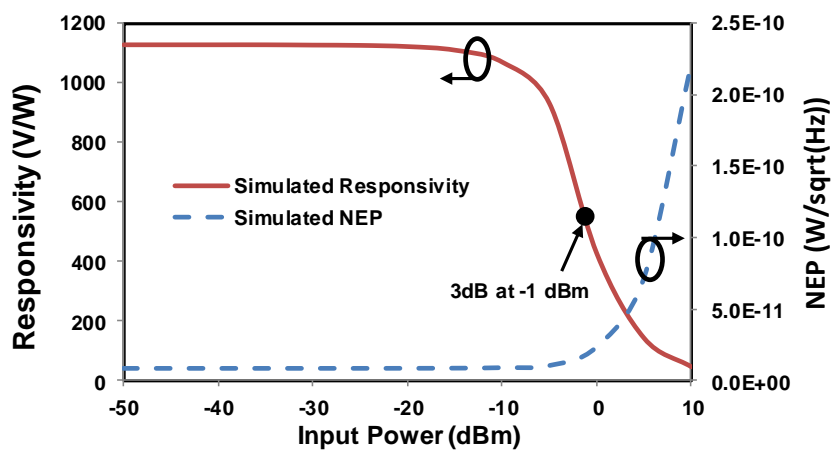

Fig. 3 Simulated responsivity (solid line) and NEP (dash line) versus input power of the CMOS detector in a $65 \mathrm{~nm}$ technology at $100 \mathrm{GHz}$.

drops with the increasing of the bias current. Therefore, the minimum NEP also occurs around the threshold voltage, shown in Fig. 2. But, a subtle difference is that responsivity has a stronger dependence on the gate source bias voltage, while the NEP has a relative flat response when close to the device threshold voltage. This is because of the drop of responsivity is offset by the noise decrease when the gate source bias voltage reduces from the device threshold. The analysis indicates that the optimum bias condition is independent to device size, circuit parameters, only affected by the device threshold voltage.

The device responsivity and NEP versus input power affects the detector deployment. Eqn. (2) predicts that the MOS detector responsivity would remain constant given an ideal square law model. Fig. 3 presents this trend with the input power up to $-20 \mathrm{dBm}$, after that, the responsivity drops due to short channel effects illustrated by Eqn. (4). Accordingly, the NEP maintains constant initially and increases with the responsivity drop. In real circuits, the responsivity may be affected further by the nonlinearity of parasitic capacitors and the voltage headroom reduction at the drain when driven by a large signal, which may happen in active imaging systems. In passive imaging systems, we treat the square root CMOS power detector having constant responsivity and NEP since the input power is normally low. For active imaging, the performance maintains well up to an input power about $-20 \mathrm{dBm}$ according to Fig. 3. The relative constant responsivity over a wide range of input power makes CMOS detectors suitable for large 
dynamic range applications, such as large field-of-view imaging systems.

\section{B. Bipolar Power Detector}

Bipolar and diode based power detectors often demonstrate a larger responsivity than CMOS power detectors due to their exponential I-V relationship. Due to their similar working mechanism, this section only uses bipolar as an example to exam this exponential I-V relation based devices, whose I-V relation can be expressed as:

$$
I_{b p}=I_{0} \exp \left(\frac{V_{B E 0}+v_{i n}}{V_{T}}\right)
$$

Accordingly, the detector responsivity is formulated as:

$$
R_{P_{-} b p}=\frac{\partial^{2} I_{b p}}{\partial v_{i n}{ }^{2}} R=\frac{I_{b p} R}{V_{T}^{2}}
$$

Eqn. (10) indicates that the bipolar detector responsivity increases with the device bias current. Figure 4 shows a detector schematic designed in an InP HBT technology, supported by HRL Laboratories [22]. The InP device peak $\mathrm{f}_{\mathrm{T}}$ is about $350 \mathrm{GHz}$ when the collector bias current is about $6 \mathrm{~mA}$ and the $\mathrm{f}_{\mathrm{T}}$ drops to about $70 \mathrm{GHz}$ with $100 \mu \mathrm{A}$ collector bias current [22]. This technology supports both InP and Silicon BiCMOS devices by intimately integrating InP HBT devices together with deep scaled CMOS devices.

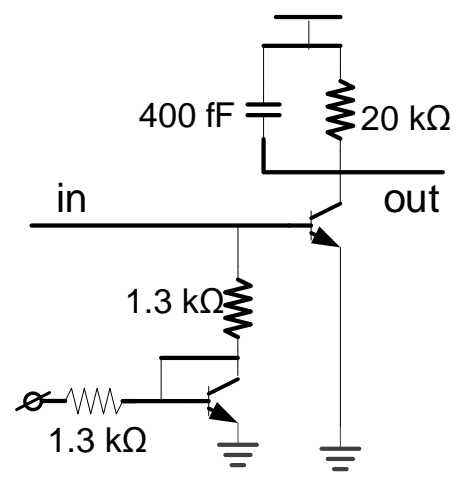

Fig. 4 A bipolar detector schematic in a $0.25 \mathrm{um} \mathrm{InP} \mathrm{HBT/Silicon}$ BiCMOS technology

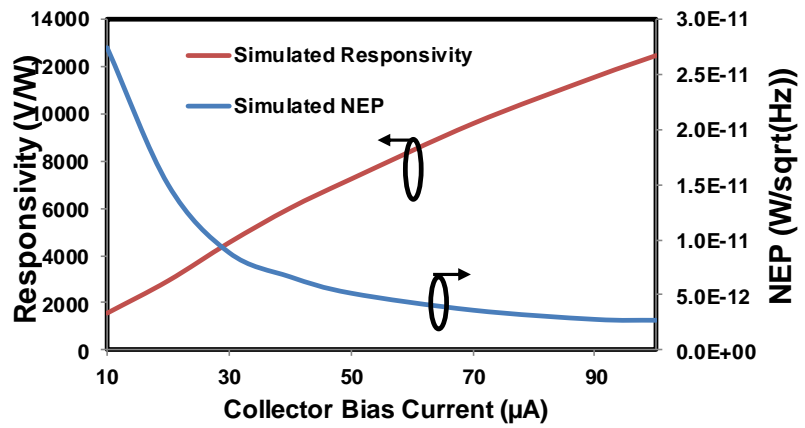

Fig. 5 Simulated responsivity (solid line) and NEP (dash line) versus collector bias current for a bipolar detector at $67 \mathrm{GHz}$ in a 0.25um InP HBT/Silicon BiCMOS technology
Fig. 5 shows that the simulated responsivity and NEP versus collector bias current with a small input power. The responsivity increases with the collector bias current, which agrees with the analysis. Similar to the analysis of CMOS power detector, the NEP of bipolar devices can be derived as the detector output noise over the responsivity:

$$
N E P_{b p}=\frac{\sqrt{4 K T R+4 K T \gamma R^{2} I_{b p} /\left(\xi V_{T}\right)}}{\left(I_{b p} /\left(\xi V_{T}\right)^{2}\right) R}
$$

Eqn. (11) suggests that the NEP of bipolar power detectors reduces with a larger bias current, which is also verified by simulation results in Fig. 5. Fig. 5 indicates that although a larger current results in a higher output noise, a larger responsivity overtakes that effect and leads to a smaller NEP. When the bias current $I_{b p}$ is small, the NEP is dominated by load resistor, represented by the first item inside the square root sign. The increasing of $I_{b p}$ leads to linear drop of NEP. With the bias current $I_{b p}$ further increasing, the noise from active device dominates, represented as the second item. Therefore, NEP reduction rate is a square root function of $I_{b p}$. Overall, with a small input power, bipolar detectors need to be biased with a larger current to achieve higher responsivity and lower NEP.

When the input power increases, Eqn. (10) suggests that the bipolar transistor based power detector responsivity increases accordingly. Fig. 6 shows the simulated responsivity and NEP versus input signal power of the bipolar detector in an InP technology, which do not change too much when the power is lower than $-30 \mathrm{dBm}$. It is because an ultra-small power does not induce significant change of collector current even with the exponential I-V relationship of the bipolar transistor.

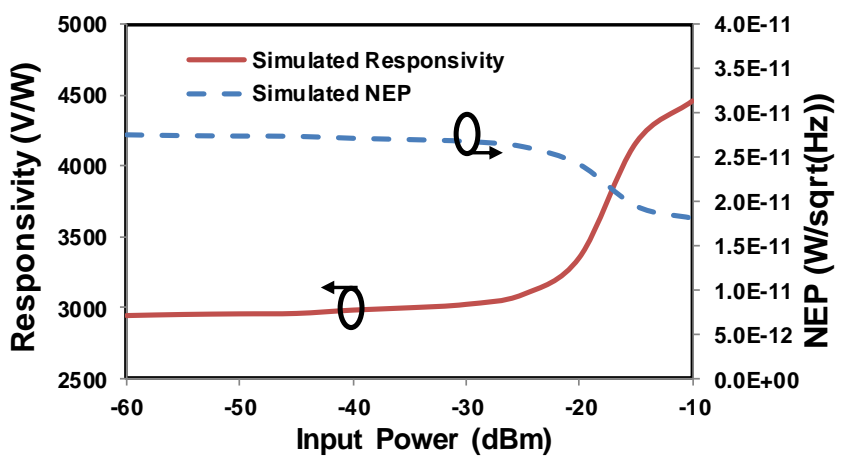

Fig. 6 The simulated responsivity (solid line) and NEP (dash line) versus input power of the bipolar detector in a $0.25 \mathrm{um} \mathrm{InP}$ HBT/Silicon BiCMOS technology at $67 \mathrm{GHz}$.
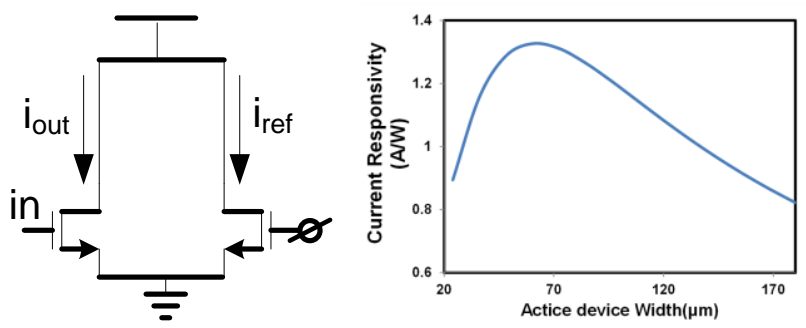

Fig. 7 The setup to simulate the current responsivity and the simulated result versus active device width. 
Therefore, we can still assume the bipolar detector has constant responsivity and NEP when the input power is low, which can be applied to passive imagers and most of active imagers. When the input power continues increasing, the responsivity increases and the NEP drops, which is consistent with the analytical results. In addition, when the input power is large, the optimum bias current drops to save the power consumption. Therefore, bipolar detectors perform better in high input power scenarios, which is different from CMOS detectors.

\section{INTEGRATION OF CMOS AND BIPOLAR DETECTORS}

Design of standalone and integrated detectors preceded by amplifiers are different. For standalone detectors, the detector NEP and bandwidth directly determine imager performance, which need to be optimized as the first priority. However, in integrated detectors, preceding amplifiers may be the dominant component for system sensitivity where the detector NEP is negligible. This paper focuses on the design for integrated systems, therefore, we skips the design optimization for standalone detectors. Eqn. (12) shows the system NETD versus circuit performance parameters [23]:

$$
N E T D=\sqrt{\left(\frac{1}{B \tau}\right) T_{S N}^{2}+\left(\frac{N E P_{\mathrm{det}}}{k G B}\right)^{2} \frac{1}{2 \tau}}
$$

where B and G are bandwidth and gain of RF front-end, $\tau$ is the system integration time, $T_{S N}$ is the system noise equivalent temperature, and $\mathrm{NEP}_{\text {det }}$ is the detector NEP following the RF front-end. When the second item is much smaller than the first item, the imager sensitivity is determined by the RF front-end. The detector NEP is negligible. To satisfy this criterion, the detector NEP and LNA output noise should meet the requirement as:

$$
\frac{N E P_{\mathrm{det}}}{2 \sqrt{B}}<\frac{1}{10} v_{n}^{2}
$$

where $v_{n}^{2}$ is the output noise of the RF front-end. The noise is equivalent at the RF front-end output node, which is also the detector input node.

The design procedure to optimize integrated CMOS and bipolar detectors are different. For CMOS detectors, since the optimum bias condition occurs when the gate source voltage biases at the device threshold voltage, which is relatively fixed. Therefore, the most sensitive component to the NEP is the active device size. At low operating frequencies, a larger device size leads to a larger bias current which therefore results in a higher responsivity and lower NEP. However, with the increasing of the operating frequency, the responsivity may not further increase with the device size due to parasitics. To meet the bandwidth requirement that having negligible detector NEP compared with the RF front end output noise, the detector size is chosen to be large enough so that the detector NEP satisfies Eqn. (13). Based on the simulation, the designed CMOS LNA output noise is about $3.6 \mathrm{e}-16 \mathrm{~V}^{2} / \mathrm{Hz}$. With a $20 \mathrm{GHz}$ front end bandwidth, the detector NEP should be smaller than 10.3 $\mathrm{pW} / \mathrm{sqrt}(\mathrm{Hz})$. The minimum device size is $35 \mu \mathrm{m} / 0.1 \mu \mathrm{m}$ based on simulation. We choose $0.1 \mu \mathrm{m}$ device length instead of 65 $\mathrm{nm}$ to reduce flicker noise. Next, we optimize the device size, while larger than the minimum size determined by Eqn. (13), to find the one corresponding to the highest current responsivity at the operating frequency. Figure 7 shows the setup to find the current responsivity and the simulation result of current responsivity versus active device size. It has been found that device width of about $60 \mu \mathrm{m}$ demonstrating the highest current responsivity. After the active device size is determined, the passive resistor load at the drain is chosen to accommodate the highest input power with $3 \mathrm{~dB}$ margin before sending the active device into the triode region. We recap the design procedure of integrated CMOS detectors as the four steps:

1. Bias the detector at an optimum voltage, close to the active device threshold voltage.

2. Identify the preceding RF front end output noise voltage and obtain the detector's minimum active device size requirement to satisfy Eqn. (13).

3. Determine the active device size to achieve maximum current responsivity based on the Fig. 7. The device size is larger than the minimum device size from step 2 .

4. Determine the maximum allowable drain load resistor value for a large responsivity, while accommodating the highest input power level with $3 \mathrm{~dB}$ margin without sending the active device into the triode region.

As for integrated bipolar detectors, the design procedure is similar to CMOS detectors but with minor adjustments. It is because the bias of bipolar device has a wider range of choice, the active device size option is mainly determined by the operation bandwidth. The design procedure is summarized as: 1. Optimize the bipolar device size to provide the widest bandwidth.

2. Determine the bias current so that the detector's NEP satisfies Eqn. (13) to be negligible compared to the RF front end output noise.

3. Maximize the load resistance $\mathrm{R}$ for a large responsivity and to support the highest possible input power with $3 \mathrm{~dB}$ margin without pushing device into triode region.

This procedure indicates the optimization method for a broadband detector, which covers the operating frequency from DC up to the highest target frequency.

\section{SUPER-REgENERATIVE Detector}

Aforementioned single device based detectors have the constraints of small responsivity which cannot efficiently suppress noises from the subsequent circuits. Conventional approaches are to add amplifiers in the front to increase the responsivity, which however suffers from high power consumption for mm-wave frequency operations.

To dramatically reduce the power consumption while still maintaining high responsivity, the authors have proposed a super-regenerative structure based detector [15, 16, 17], which leverages ultra-high quality factor of super-regenerative amplifiers for high responsivity. In essence, the super-regenerative detector merges a super-regenerative amplifier with an envelope detector, as shown in Fig. 8(a). The super-regenerative amplifier (SRA) amplifies the signal, whose envelope is then extracted by the detector. It is equivalent to an imaging sub-system that has both amplifiers and detectors while maintaining ultra-low power and high-frequency operations. 


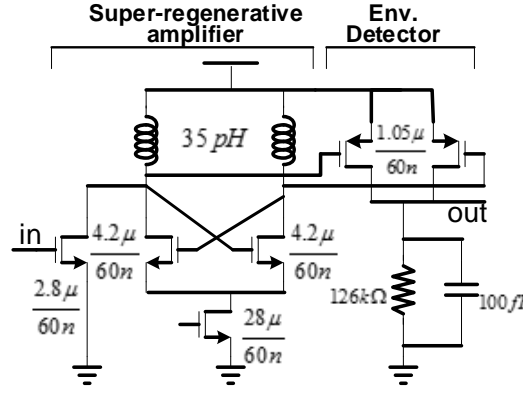

(a)

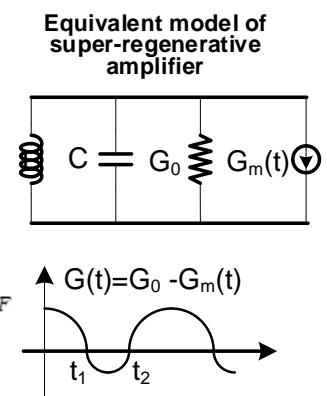

(b)
Fig. 8 (a) The schematic of super-regenerative detector in a $65 \mathrm{~nm}$ CMOS technology, (b) the equivalent mode of the super-regenerative amplifier and the tank overall conductance change controlled by the quench signal.

A super-regenerative amplifier is based on a quench signal controlled oscillator, as shown in Fig. 8(a). The equivalent circuit model can be illustrated in Fig. 8(b). The overall transconductance is time-variant, and can be represented as [24]:

$$
G(t)=G_{0}-G_{m}(t)
$$

where $G_{0}$ is the equivalent conductance of the passive resonator at the resonant frequency $\omega_{0},-G_{m}(t)$ is the instantaneous trans-conductance generated by the cross-coupled pair which is controlled by the quench signal applied to the bottom current source device. With a periodic quench signal, $G(t)$ is also periodic, varying from positive to negative and back to positive periodically. Based on the output signal amplitude, super-regenerative amplifier can be categorized into linear mode and logarithmic mode. In the linear mode operation, the super-regenerative amplifier output amplitude is linearly proportional to input signal amplitude. In the logarithmic mode, the super-regenerative amplifier output signal eventually reaches a saturation voltage before quenching down to a $\mathrm{DC}$ at the end of each cycle.

A typical $G(t)$ under the linear mode operation is sketched in Fig. 8(b). The circuit can demonstrate ultra-sensitive responses during the transition region of the conductance value changing from positive to negative, annotated as $t_{1}$ in the figure. The voltage gain is proportional to the slope of the $G\left(t_{1}\right)$, i.e. $G^{\prime}\left(t_{1}\right)$. Therefore, the total voltage gain of a super regenerative amplifier can be formulated as [24]:

$$
\begin{aligned}
& A_{V}=s(t) p(t) u(t) \\
& =G_{0} \sqrt{\frac{\pi}{C\left|G^{\prime}\left(t_{1}\right)\right|}} \frac{\omega}{\omega_{0}} \exp \left(\frac{C\left(\omega-\omega_{0}\right)^{2}}{-\left|G^{\prime}\left(t_{1}\right)\right|}\right) \exp \left(\frac{-1}{2 C} \int_{t_{1}}^{t} G(\tau) d \tau\right)^{(15)}
\end{aligned}
$$

Where $s(t)$ is the gain factor, $\mathrm{p}(\mathrm{t})$ is the bandwidth factor, and $\mathrm{u}(\mathrm{t})$ is the super-regenerative factor, can be represented respectively as:

$$
\begin{aligned}
& s(t)=G_{0} \sqrt{\frac{\pi}{C\left|G^{\prime}\left(t_{1}\right)\right|}}, p(t)=\frac{\omega}{\omega_{0}} \exp \left(\frac{C\left(\omega-\omega_{0}\right)^{2}}{-\left|G^{\prime}\left(t_{1}\right)\right|}\right) \\
& u(t)=\exp \left(\frac{-1}{2 C} \int_{t_{1}}^{t} G(\tau) d \tau\right)
\end{aligned}
$$

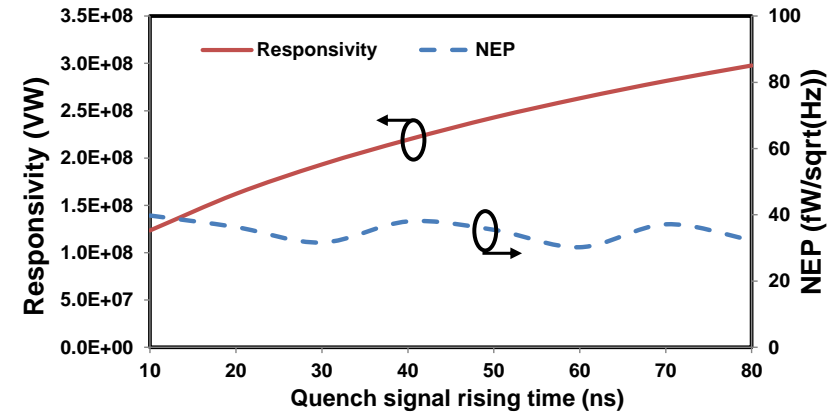

Fig.9 The simulated responsivity and NEP versus the quench rising time

where $\mathrm{C}$ is the overall capacitance of the resonant tank. The bandwidth of a super-regenerative amplifier is narrow due to its Q-boosting characteristics. The signal voltage envelope can be expressed as:

$$
V_{\text {env }}=V_{\text {in }} A_{V}
$$

Following the super-regenerative amplifier, a CMOS detector extracts the envelope information as the imagers in $[15,16]$. Since the detector output DC voltage $\left(V_{\text {det }}\right)$ can be derived from the input voltage with the relation of $V_{\text {det }}=$ $K_{\text {det }}\left(V_{\text {env }}\right)^{2}$, the total responsivity of the super-regenerative amplifier in linear mode together with the detector can be presented as:

$$
R_{p_{-} \text {det }}=2 K_{\text {det }} A_{V}^{2}
$$

The super-regenerative amplifier magnifies the noise with the same ratio as the input signal. Therefore, the input referred noise is similar to conventional amplifiers. Although the noise performance of super-regenerative amplifiers is similar to that of conventional amplifiers, its large responsivity suppresses subsequent circuit noise significantly, which may not be trivial at the same power efficiency by conventional amplifiers.

Figure 9 presents the detector responsivity versus quench signal rising time, which is inversely proportional to the slope. The simulation results suggest that the responsivity is proportional to the square root of the quench rising time, consistent with the analysis by Eqn. (15) [24]. However, the achieved NEP is similar with different quench signal slopes, which is mainly due to the relatively constant input referred noise.

Above analysis describes the operation of linear mode super-regenerative detectors. For logarithmic mode operation, the output envelope does not linearly follow the input amplitude. Instead, the output signal builds up to the oscillator saturation amplitude exponentially. The relationship of the oscillator start-up time and the input voltage amplitude can be expressed as [25]:

$$
t=\tau_{0} \ln \left(\frac{V_{T H}}{v_{i n}}\right)
$$

where the time $\tau_{0}$ is a time constant related to the oscillator parameters and inversely proportional to the super-regenerative gain $A_{V}$, and $V_{T H}$ is the threshold voltage to turn on the switches to generate pulses. Therefore, the pulse width is proportional to $t$ defined by Eqn. (19). The responsivity, 
defined by the equivalent DC voltage change versus the input power, can be presented as following:

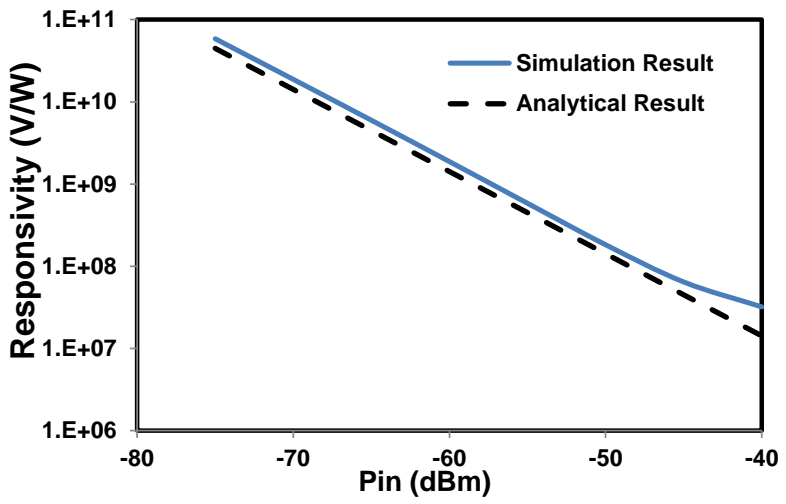

Fig. 10 The analytical (dash line) and simulated (solid line) responsivity versus input power of a super-regenerative detector at $200 \mathrm{GHz}$.

$$
R_{P_{-} S R}=\frac{\partial^{2} t}{\partial v_{i n}{ }^{2}} V_{D D}=\frac{t_{0}}{v_{i n}{ }^{2}} V_{D D} \propto \frac{1}{p_{i n}}
$$

where $V_{D D}$ is the power supply, which is $1 \mathrm{~V}$ for a $65 \mathrm{~nm}$ CMOS technology. Fig. 10 shows simulated and analytical responsivity versus input signal power, which verifies the inversely proportional relation to the input power, predicted by Eqn. (20).

It is worthy to mention that due to the large gain of the super-regenerative amplifier, both NEP and responsivity are determined by the super-regenerative amplifier while not the subsequent detector.

According to Eqn. (15), the 3-dB bandwidth of a super-regenerative amplifier can be derived as:

$$
B_{S_{-} 3 d B}=\frac{3 \ln 10}{20 \pi} \sqrt{\frac{\left|G^{\prime}\left(t_{1}\right)\right|}{C}}
$$

Lowering down the $G^{\prime}\left(t_{1}\right)$ increases the signal gain, as shown in Eqn. (15), however, at the cost of a smaller bandwidth. For a conventional amplifier with the same resonant tank as the load, the bandwidth can be calculated as

$$
B_{\text {conv_ } 3 d B}=\frac{\omega_{0}}{Q}=\frac{G_{0}}{2 \pi C}
$$

Therefore, the bandwidth ratio between a super-regenerative amplifier and a conventional amplifier is proportional to $\sqrt{G^{\prime}\left(t_{1}\right) / C}$.

The key circuit parameter to determine super-regenerative detectors' performance is the quench signal rising time, as shown in Fig. 9, not the bias condition like CMOS and bipolar detectors. This enables extreme low power consumption circuits to achieve ultra-high responsivity, and is applicable to power sensitive applications, such as embedded and biological applications. The responsivity increases with the drop of input power makes this type of detectors suitable for ultra-low power application scenarios.

\section{MEASUREMENT RESULTS AND COMPARISON SUMMARY}
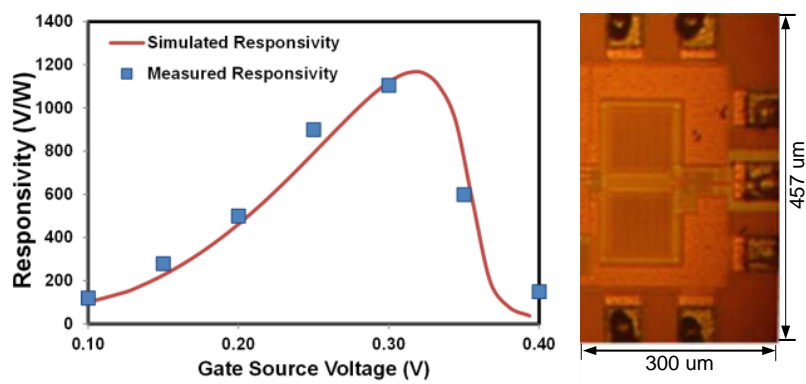

Fig. 11 Responsivity versus the gate bias voltage at $100 \mathrm{GHz}$ for both simulated (line) and measured (dots) results of a CMOS detector in a $65 \mathrm{~nm}$ technology and its die photo

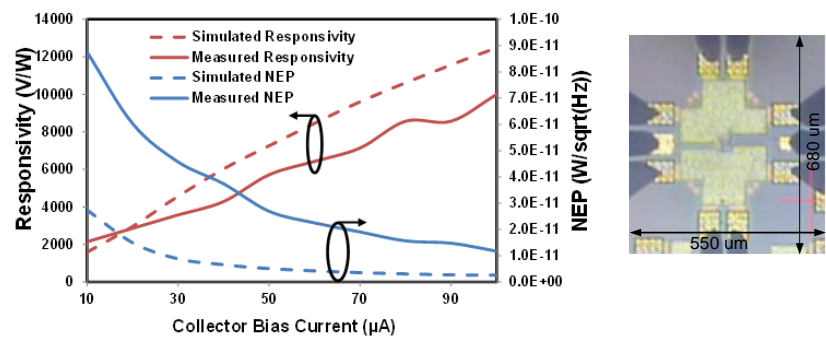

Fig. 12 Measured responsivity and NEP (solid lines) versus collector bias current compared with simulation results (dash lines) of a bipolar detector in a $0.25 \mathrm{um} \mathrm{InP} \mathrm{HBT} \mathrm{technology} \mathrm{and}$ its die photo.

\section{A. Measurement Results}

Figure 11 shows the die photo of an integrated CMOS detector in a $65 \mathrm{~nm}$ CMOS technology and the measured and simulated responsivity versus the gate source voltage. A monolithic multiplier chain generates the input signal with an attenuator to adjust the input power level. The output voltage is measured by an oscilloscope. The measured responsivity demonstrates the peak around the device threshold region, which is consistent with analytical and simulation results. Because the detector follows a preceding LNA, its NEP cannot be measured individually because the imager NEP is determined by the LNA. The detector core chip size is $220 \mu \mathrm{m}$ $\mathrm{X} 110 \mu \mathrm{m}$.

Figure 12 shows the measured responsivity and NEP versus the collector bias current of a bipolar detector in a $0.25 \mathrm{um} \mathrm{InP}$ HBT technology provided by HRL Laboratories, with the simulated results included for the comparison. In this measurement setup, Agilent E8267D generates the input signal. The output DC voltage is measured by Keithley 2400 source meter and the noise floor is measured by a spectrum analyzer. This is measured when the input power is $-50 \mathrm{dBm}$. It shows that the responsivity increases with bias current, while the NEP drops with bias current. The core chip area is $100 \mu \mathrm{m} \mathrm{X} 25 \mu \mathrm{m}$. Fig. 13(a) presents the measured and simulated responsivity and NEP versus input power at $20 \mu \mathrm{A}$ collector bias current, demonstrating that the responsivity increases and the NEP drops with input power increasing, which is consistent with the analytical results. Fig. 13(b) presents the responsivity versus input power at different collector current bias conditions, $20 \mu \mathrm{A}$ and $40 \mu \mathrm{A}$. At $20 \mu \mathrm{A}$ condition, the responsivity increases with 
input power which is consistent with analytical results due to the equivalent larger bias current boosted by the input signal. At $40 \mu \mathrm{A}$ condition, the responsivity first increases with input power due to the same reason. But, after reaching a peak value, the responsivity then drops because the active device is driven into the triode region due to a large current flowing through the collector load resistor. In addition, the bipolar detector also shows different optimum bias for different input power. When with a small input power, the bias current needs to be large. When the input power increases, the corresponding optimum bias current should reduce to avoid pushing the device into the triode region. In practical circuits, the optimum bias current is also affected by the supply voltage and the load resistance value. The larger the supply voltage and the smaller the load resistance, the higher the optimum bias current is. Therefore, the optimum bias current of the bipolar detector depends on not only the device characteristics, but also the input signal power, which demonstrates non-constant responsivity and NEP and is different from CMOS power detectors.

Figure 14 shows the super-regenerative detector chip photo
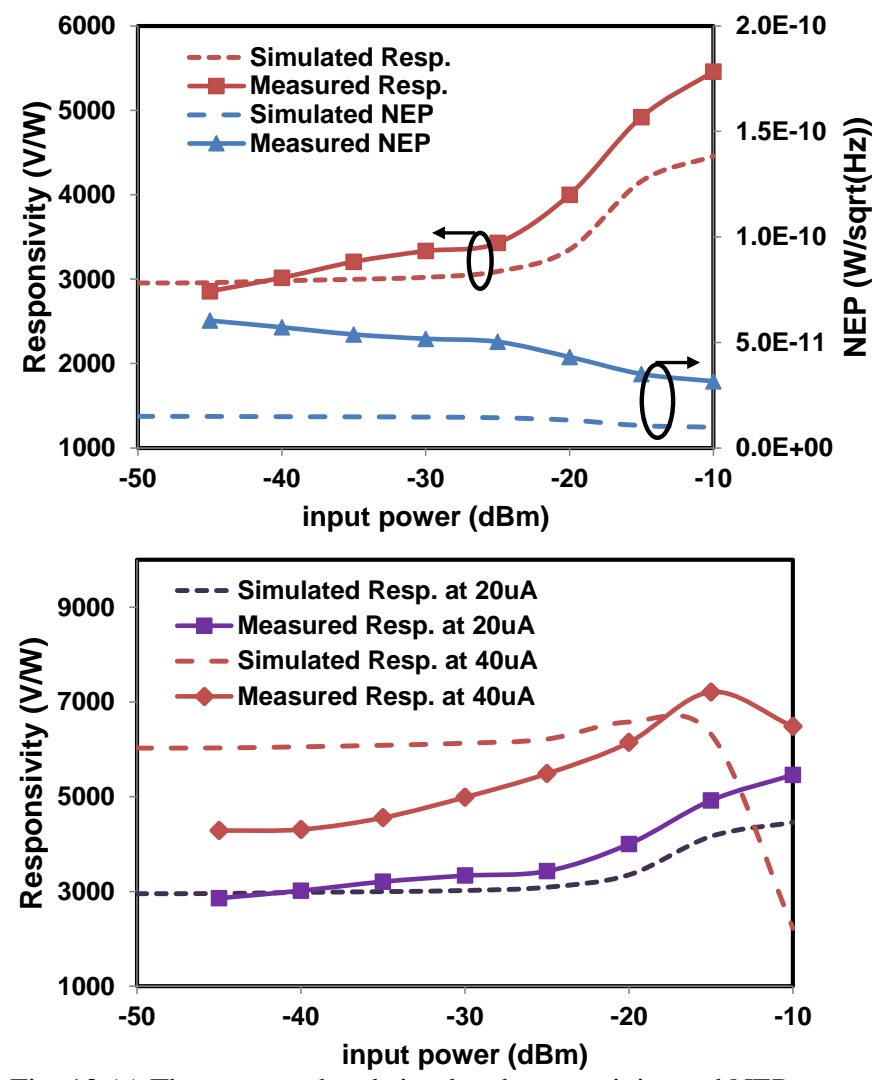

Fig. 13 (a) The measured and simulated responsivity and NEP versus input power at $20 \mu \mathrm{A}$ collector bias current, and (b) the measured and simulated responsivitv versus input power at different collector bias

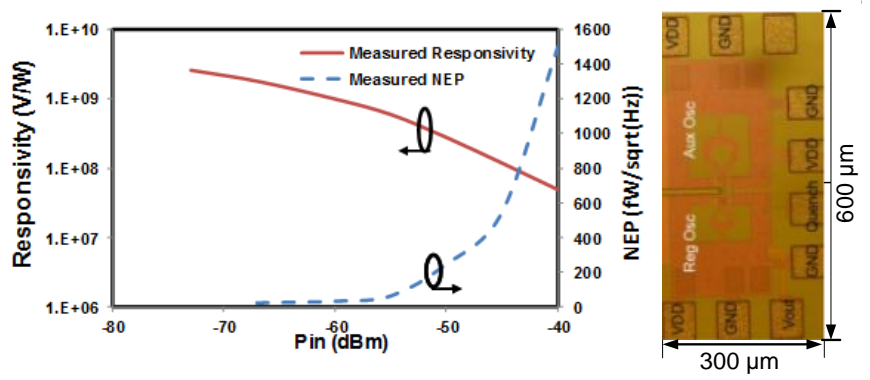

Fig. 14 The measured responsivity (solid line) and NEP (dash line) versus input power for a super regenerative detector in a $65 \mathrm{~nm}$ CMOS technology at $200 \mathrm{GHz}$ fabricated in a $65 \mathrm{~nm}$ CMOS technology and the measured responsivity and NEP. It uses an AMC (active multiplier chain) from VDI Inc. as the $200 \mathrm{GHz}$ source with an attenuator and a power meter to characterize the power level. The output voltage of detector is recorded with a data-acquisition module and the noise floor is measured with a spectrum analyzer. The detail measurement setup is illustrated and explained in [16]. The core chip size is $240 \mu \mathrm{m}$ X $100 \mu \mathrm{m}$. The responsivity drops with input power dramatically, which is consistent with the analytical and simulation results presented in Section V. NEP is therefore increasing with the input power. It suggests that super-regenerative detectors have superior responsivity and sensitivity, particularly suitable for low power applications.

\section{B. Comparison Summary}

Table 1 summarizes the key differences among the three types of detectors, including performance comparison in responsivity, NEP, and bandwidth, key circuit parameters to determine each detector's performance, detectors' response and optimization with regarding to the input power level, and the defined $\mathrm{FOM}_{\mathrm{P}}$ and $\mathrm{FOM}_{\mathrm{A}}$ to provide quantitative comparison for each application scenario. Due to no measured NEP of the CMOS detector, simulation results are used instead. All other numbers are based on measurement results.

High responsivity is beneficial to suppress the noise of subsequent circuits. A super regenerative detector presents the highest responsivity, followed by a bipolar detector, and then a CMOS detector. This is because the super-regenerative detector essentially adds an amplifier in the front, and the bipolar detector has a larger non-linearity than the CMOS detector to obtain a larger conversion rate from the input power to the output voltage. In terms of NEP, CMOS detectors' NEPs are relatively large mainly due to the device flicker noise and

\begin{tabular}{|c|c|c|c|}
\hline & $\begin{array}{l}\text { CMOS } \\
\text { Detector }\end{array}$ & $\begin{array}{l}\text { Bipolar } \\
\text { Detector }\end{array}$ & $\begin{array}{l}\text { Super Reg. } \\
\text { Detector }\end{array}$ \\
\hline Responsivity & $\begin{array}{l}\text { Medium; Optimum } \\
\text { bias around device } \\
\text { threshold voltage }\end{array}$ & \begin{tabular}{|l|} 
Large; Optimum \\
bias affected by \\
the input signal \\
power
\end{tabular} & $\begin{array}{|lr|}\text { Super } & \text { Large; } \\
\text { Perfromance } & \text { is } \\
\text { determined by the } \\
\text { quench signal slope } \\
\end{array}$ \\
\hline NEP & $\begin{array}{l}\text { Large due to large } \\
\text { flicker noise and } \\
\text { small responsivity }\end{array}$ & \begin{tabular}{|l|} 
Medium due to \\
large responsivity \\
with a large bias \\
current
\end{tabular} & \begin{tabular}{|l} 
Small due to the \\
front \\
regenerative amplifier
\end{tabular} \\
\hline Bandwidth & $\begin{array}{l}\text { Wide from DC up } \\
\text { to device cutoff } \\
\text { frequency }\end{array}$ & $\begin{array}{l}\text { Wide from DC up } \\
\text { to device cutoff } \\
\text { frequency }\end{array}$ & $\begin{array}{l}\text { Small due to high Q } \\
\text { of the oscillator }\end{array}$ \\
\hline $\begin{array}{l}\text { Responsivity } \\
\text { vs input } \\
\text { power }\end{array}$ & $\begin{array}{l}\text { Constant, } \\
\text { slightly decrease } \\
\text { due to short } \\
\text { channel effects }\end{array}$ & \begin{tabular}{|l|}
$\begin{array}{l}\text { Increase } \\
\text { input power }\end{array}$ \\
\end{tabular} & $\begin{array}{l}\text { Decrease with input } \\
\text { power }\end{array}$ \\
\hline FOM $_{P}$ & $254^{*}$ & 250 & 231 \\
\hline $\mathrm{FOM}_{\mathrm{A}}$ & $144^{*}$ & 142 & 158 \\
\hline
\end{tabular}

$*$ CMOS detector NEP number comes from simulation due to no measurement results

intrinsically small responsivity. Bipolar detectors' NEP can be reduced by increasing the bias current to increase responsivity and suppress output noise to a certain extent. The NEP of 
super-regenerative detectors is often better than the previous two because they have an amplifier in the front. Within super-regenerative detectors, CMOS implementations may present a larger NEP than Bipolar based ones due to their high noise figure performance. With regard to the achievable bandwidth, super-regenerative detectors support a very narrow bandwidth due to the high $\mathrm{Q}$ feature of the oscillator based structure. CMOS and Bipolar detectors can be designed with a very wide bandwidth, theoretically from DC all the way up to device cutoff frequencies.

Another key difference among the three detectors is the responsivity trend versus input power. The responsivity of CMOS detectors maintains relatively constant due to the square law model I-V relation and presents slightly downward trend because of short channel effects. Bipolar detectors' responsivity in principle increases with the input power. A super-regenerative detector responsivity on the other hand drops linearly with the input power. These interesting characteristics could determine the optimum application scenarios for each detector. For example, CMOS power detectors deliver the largest dynamic range, which therefore are appropriate for the applications requiring large dynamic range conditions, such as large field-of-view imaging. However, the larger flicker noise may mandate extra calibration schemes, such as Dicke switch based imaging architecture [6, 7, 10-11]. Bipolar based detectors are more suitable for high power conditions due to the increase of responsivity with input signal power. Super-regenerative detectors are suitable for extremely low input power conditions because its responsivity actually increases when the input becomes weaker, which is also helped by the ultra-high sensitivity.

This paper intends to quantify the comparison of the three detectors and provide insight of their deployment in practical systems. Therefore, we define two FOMs: FOM $_{P}=10$ * $\log \left(\frac{B W}{N E P * \text { Power }}\right)$ for passive imaging applications and $F O M_{A}=$ $10 * \log \left(\frac{1}{N E P * \text { Power }}\right)$ for active imaging applications. Here responsivity is not included because in integrated imaging systems, the overall system responsivity is determined by the front end LNA. FOM $\mathrm{P}_{\mathrm{P}}$ of both CMOS and bipolar detectors are much higher than super-regenerative detectors, which indicates that they are suitable for passive imaging due to their wide bandwidth responses. However, super-regenerative detectors' $\mathrm{FOM}_{\mathrm{A}}$ is much higher than the other two detectors. Therefore super-regenerative detectors provide more advantages in active imaging applications. In addition, their ultra-high responsivity helps to suppress the subsequent baseband circuit noise to provide a better overall system NEP. The ultra-low power consumption of super-regenerative detectors makes it an excellent candidate in many biologic imaging applications.

\section{CONCLUSION}

This paper compares three different power detectors for integrated microwave/mm-wave imaging systems: CMOS power detectors, bipolar power detectors, and super-regenerative detectors, based on theoretical analysis of their working mechanisms. This paper for the first time presents their different responses to input power, provides design procedure for integrated CMOS and bipolar detectors and defines two FOMs for both passive and active imaging systems to quantify their capabilities for different applications. Simulations and experiments have been conducted to validate the comparison. In summary, CMOS and bipolar power detectors can support wide bandwidth operations, which however, typically needs a wideband low noise amplifier in the front and consumes large power. On the other hand, super-regenerative detectors deliver a narrow band operation but with extremely low power consumption. Since there is already an amplifier in the front, super regenerative detector can form a very compact imaging system to be applicable to a variety of applications in active imaging systems, such as biological diagnosis.

\section{ACKNOWLEDGMENT}

The authors would like to thank DARPA COSMOS Program and Dr. Sanjay Raman for funding support.

\section{REFERENCES}

[1] Z. Wang, P.-Y. Chiang, P. Nazari, C.-C. Wang, Z. Chen, and P. Heydari, "A CMOS $210 \mathrm{GHz}$ Fundamental Transceiver with OOK Modulation," IEEE Journal of Solid-State, vol. 49, no. 3, pp. 564-580, March 2014

[2] S. Moghadami, F. Hajilou, P. Agrawal, and S. Ardalan "A 210 $\mathrm{GHz}$ Fully-Integrated OOK Transceiver for Short-Range Wireless Chip-to-Chip Communication in 40nm CMOS Technology," IEEE Transactions on Terahertz Science and Technology, vol. 5, no. 5, pp. 737-741, Sep. 2015

[3] J. D. Park, S. Kang, S.V. Thyagarajan, E. Alon, and A.M. Niknejad, "A $260 \mathrm{GHz}$ Fully Integrated CMOS Transceiver for Wireless Chip-to-Chip Communication," Symposium on VLSI Circuits, pp. 48-49, June 2012

[4] Q. Fang, P.M. Meaney, and K.D. Paulsen, "Viable Three-Dimensional Medical Microwave Tomography: Theory and Numerical Experiments," IEEE Trans Antennas Propag, vol. 58, (no. 2), pp. 449-458, Feb 12010

[5] Meaney, P. M., M. W. Fanning, D. Li, S. P. Poplack, and K. D. Paulsen, "A clinical prototype of active microwave imaging of the breast," IEEE Transactions on Microwave Theory and Techniques, Vol. 48, 1841-1853, 2000

[6] A. Tomkins, P. Garcia, and S. P. Voinigescu, "A Passive W-Band Imaging Receiver in 65-nm Bulk CMOS," IEEE J. of Solid-State Circuits, vol. 45, no. 10, pp. 1981-1991, Oct. 2010.

[7] Q. J. Gu, K. Yang, Y. Xue, Z. Xu, A. Tang, C. C. Nien, T. H. Wu, J. H. Tarng and M.-C. F. Chang, "A CMOS Integrated W-Band Passive Imager," IEEE Transactions on Circuits and Systems II, vol. 59, no. 11, pp. 736-740, Nov. 2012

[8] D. Öjefors, U. Pfeiffer, A. Lisauskas, and H. G. Roskos, “A 0.65 $\mathrm{THz}$ focal-plane array in a quarter-micron CMOS process technology", IEEE Journal of Solid-State Circuits, vol. 44, no. 7, pp. 1968-1976, July 2009

[9] R. Han, Y. Zhang, D. Coquillat, H. Videlier, W. Knap, E. Brown, and K. K. O, "A 280-GHz Schottky Diode Detector in 130-nm Digital CMOS," IEEE Journal of Solid-State Circuits, vol. 46, no. 11, pp. 2602-2612, November 2011

[10] L. Gilreath, V. Jain, H.-C. Yao, L. Zheng, and P. Heydari, "A 94-GHz Passive Imaging Receiver using a Balanced LNA with Embedded Dicke Switch," 2010 IEEE Radio Frequency Integrated Circuits Symposium

[11] J. W. May, and G. M. Rebeiz, "Design and Characterization of W-Band SiGe RFICs for Passive Millimeter-Wave Imaging," 
IEEE Transactions on Microwave Theory and Techniques, vol. 58, no. 5, pp. 1420-1430, May 2010

[12] J. J. Lynch, P. A. Macdonald, H. P. Moyer, and R. G. Nagele, "Passive millimeter wave imaging sensors for commercial markets," Applied Optics, vol. 49, no. 19, pp. E7-e12, July 2010

[13] J. J. Lynch, H. P. Moyer, J. H. Schaffner, Y. Royter, M. Sokolich,B. Hughes, Y. J. Yoon, and J. N. Schulman, "Passive millimeter-wave imaging module with preamplified zero-bias detection," IEEE Transactions Microwave Theory Tech. vol. 56, no.7, pp. 1592-1600, July 2008.

[14] H. P. Moyer, J. N. Schulman, J. J. Lynch, J. H. Schaffner, M. Sokolich, Y. Royter, R. L. Bowen, C. F. McGuire, M. Hu, and A. Schmitz, "W-Band Sb-Diode Detector MMICs for Passive Millimeter Wave Imaging," IEEE Microwave and Wireless Components Letters, vol. 18, no. 10, October 2008

[15] A. Tang, Q. Gu, Z. Xu, V. Gabriel, M.C. F. Chang, "A max 349 $\mathrm{GHz} 18.2 \mathrm{~mW} /$ pixel CMOS inter-modulated regenerative receiver for tri-color mm-Wave imaging," IEEE MTT International Microwave Symposium, May 2012

[16] A. Tang and M.-C. Frank Chang, "Inter-Modulated Regenerative Receivers Operating at $349 \mathrm{GHz}$ and $495 \mathrm{GHz}$ for THz Imaging in 40nm and $65 \mathrm{~nm}$ CMOS technology", IEEE Transactions on Terahertz Science and Technology, Vol 3, No 2, pp 134-140, March 2013

[17] Tang, A.; Chang, M.-C.F., "183GHz 13.5mW/pixel CMOS regenerative receiver for mm-wave imaging applications," IEEE International Solid-State Circuits Conference, 2011, pp. 296-297, Feb. 2011

[18] J. L. Bohorquez, A. P. Chandrakasan, and J. L. Dawson, "Frequency-Domain Analysis of Super-Regenerative Amplifiers," IEEE Transactions on Microwave Theory and Techniques, vol. 57, no. 12, pp. 2882-2894, December 2009

[19] B. Otis, Y.H. Chee, and J. Rabaey, "A 400 uW-Rx, 1.6mW-Tx Super-Regenerative Transceiver for Wireless Sensor Networks," IEEE ISSCC pp.396-397, February 2005

[20] A. Tang, G. Virbila, D. Murphy, F. Hsiao, Y.H. Wang, Q.J. Gu, Z. $\mathrm{Xu}, \mathrm{Y}$. $\mathrm{Wu}, \mathrm{M}$. Zhu, M.C.F. Chang, "A $144 \mathrm{GHz} 0.76 \mathrm{~cm}$ resolution sub-carrier SAR phase radar for 3D imaging in $65 \mathrm{~nm}$ CMOS," ," IEEE International Solid-State Circuits Conference, pp. 265-266, Feb. 2012

[21] Y. Shang, H. Yu, S. Hu, Y. Liang, X. Bi, and M. A. Arasu, "High-sensitivity CMOS Super-regenerative Receiver with Quench-controlled High-Q Metamaterial Resonator for Millimeter-wave Imaging at 96 and $135 \mathrm{GHz}$, IEEE Transactions on Microwave Theory and Techniques, vol.62, no.12, pp. 3095-3106, December 2014

[22] J. C. Li, K. R. Elliott, D. S. Matthews, D. A. Hitko, D. Zehnder, Y. Royter, P. R. Patterson, T. Hussain, J. F. Jensen, " $100 \mathrm{GHz}+$ Gain-Bandwidth differential amplifiers in a wafer scale heterogeneously integrated technology using $250 \mathrm{~nm}$ InP DHBTs and $130 \mathrm{~nm}$ CMOS," IEEE Journal of Solid-State Circuits, vol. 44, pp. 2663-2670, Oct. 2009

[23] H. P. Moyer, J. J. Lynch, J. N. Schulman, R. L. Bowen, J. H. Schaffner, A. K. Kurdoghlian, and T. Y. Hsu, "A low noise chipset for passive millimeter wave imaging," IEEE MTT International Microwave Symposium, 2007

[24] G.G. MacFarlane and J.R. Whitehead, "The Theory of the Super-Regenerative Receiver Operated in the Linear Mode," J. of Institution of Electrical Engineers- Part III: Radio and Communication Engineering, vol. 95, pp. 143-157, May 1948

[25] F.Moncunill-Geniz, P. Pala-Schonwalder, O. Mas-Casals, "A Generic Approach to the Theory of Super-regenerative Reception" IEEE Transactions on Circuits and Systems I, vol 52, no 1, pp.54-70 Jan. 2005 